\title{
Perfil de clientes submetidos a amputações relacionadas ao diabetes mellitus
}

\author{
Profile of patients submitted to amputation related to diabetes mellitus \\ Perfíl del paciente sometido a la amputación relacionada a el diabestes mellitus
}

\begin{abstract}
Darlene Mara dos Santos Tavares', Flávia Aparecida Dias', Luciana Rabelo Araújo', Gilberto Araújo Pereira'

'Universidade Federal do Triângulo Mineiro. Centro de Graduação em Enfermagem,

Departamento de Enfermagem em Educação e Saúde Comunitária. Uberaba, MG
\end{abstract}

Submissão: 12/12/2008

Aprovação: 2 1/09/2009

\section{RESUMO}

Estudo retrospectivo Que objetivou descrever as características sócio-demográficas e clínicas de I4l sujeitos, internados no Hospital de Clínicas da Universidade Federal do Triângulo Mineiro, submetidos à amputação relacionada ao diabetes; comparar o número de amputações de acordo com as variáveis sexo, faixa etária, co-morbidades e tipo de tratamento e associar o número de amputações com o tempo de internação e de diagnóstico. Os dados coletados em prontuários totalizaram 208 amputações. Maior percentual de sujeitos do sexo masculino (58,9\%) e maior ocorrência de amputação entre os idosos (70,2\%); principal causa de internação: pé diabético (35,7\%); a maioria $(75,0 \%)$ foi internada até duas vezes; co-morbidade mais freQüente: hipertensão arterial $(74,0 \%)$; a mediana do número de amputações foi duas. Não foi observada diferença significativa entre as variáveis estudadas.

Descritores: Educação em enfermagem; Diabetes mellitus; Amputação.

\begin{abstract}
Retrospective study that aimed at describing the clinic and socio- demographical characteristics of 141 individuals which were interned in Teaching Hospital of Universidade Federal do Triângulo Mineiro, Uberaba, MG, Brazil, who were submitted to amputations related to diabetes and compare the number of amputations considering the variables sex, age, co-morbidity, the type of treatment and then associated the number of amputations with the duration of internments and diagnosis. Data were collected from patient health records, totalizing 208 amputations. Major percentage of masculine individuals $(58,9 \%)$, and a major occurrence of amputation among the elderly (70.2\%); the cause: diabetic foot (35.7\%); the most of patients (75.0\%) went through internment at least twice; most freeuent co-morbidity: arterial hypertension (74.0\%); median in the number of amputations was two. It was not observed any significant difference between the variables of study.
\end{abstract}

Descriptors: Education, nursing; Diabetes mellitus; Amputation.

\section{RESUMEN}

Estudio retrospectivo que tuvo como objetivo describir las características sociodemográficas y las clínicas de $14 \mathrm{I}$ individuos internados en el Hospital de Clínicas de la Universidade Federal do Triângulo Mineiro, Uberaba, MG, Brasil, sometidos a amputaciones relacionadas con diabetes y comparar el número de amputaciones a partir de las variables sexo, edad, comorbilidad y tipo de tratamiento, así como asociar el número de amputaciones con el tiempo de internación y de diagnóstico. Los datos fueron recolectados en las fichas médicas, totalizando 208 amputaciones. Quedó comprobado un mayor porcentual de pacientes del sexo masculino $(58,9 \%)$ y una mayor incidencia de amputaciones entre los ancianos (70,2\%); el principal causa de internación fue: pie diabético (35,7\%); la mayoría $(75,0 \%)$ fue internado dos veces; comorbilidad más frecuente: hipertensión arterial (74,0\%); promedio de amputaciones fue dos. No fueron observadas diferencias significativas entre las variables estudiadas.

Descriptores: Educación en enfermería; Diabetes mellitus; Amputación. 


\section{INTRODUÇÃO}

A prevalência de úlceras nos pés atinge $4 \%$ a $10 \%$ dos sujeitos diabéticos. De 40\% a 60\% das amputações, não traumáticas de membros inferiores, ocorrem em diabéticos, sendo Que $85 \%$ destas são precedidas de úlceras nos pés ${ }^{(1)}$. O trauma externo precipita Quatro em cada cinco úlceras nos pés de sujeitos diabéticos. A incidência de amputações relacionadas ao diabetes atinge 6-8/1000 diabéticos por ano ${ }^{(1)}$. No Brasil, estima-se Que ocorram 40.000 amputações por ano em sujeitos diabéticos ${ }^{(2)}$.

Estudo realizado no Rio de Janeiro, no período de 1990-2000, obteve taxa de incidência de amputações em membros inferiores em diabéticos 4,6 vezes maior entre sujeitos na faixa etária 55 a 74 anos $(92,19 / 100.000 \mathrm{hab})$ por ano Quando comparados com aQueles com 30 a 54 anos (20, I/ I00.000 hab). Destaca-se Que $40,1 \%$ das amputações foram localizadas na região supragenicular ${ }^{(3)}$.

Apesar dos estudos apresentarem maior prevalência do pé diabético no sexo masculino com idade acima de 58 anos $^{(4-6)}$, não foi demonstrada a relação entre o diabetes mellitus e sexo ${ }^{(6)}$.

O pé diabético se constitui em uma das complicações do diabetes mellitus e guarda relação com o tempo de duração desta doença ${ }^{(5)}$. Em longo prazo, os índices glicêmicos aumentados podem causar lesão em muitos tecidos, decorrendo de alterações micro e macrovasculares Que levam a disfunção, dano ou falência de vários órgãos ${ }^{(1)}$.

Os fatores de risco para o desenvolvimento do pé diabético estão relacionados aos antecedentes de úlceras e amputações não traumáticas nos pés; educação terapêutica deficiente; descontrole metabólico, obesidade, idade, sexo, tempo de evolução do diabetes, dificuldade de acesso ao sistema de saúde, neuropatia com diminuição de sensibilidade e deformidades; calosidades; uso de calçados inadequados; tabagismo, dentre outros ${ }^{(7)}$.

A demora no início do tratamento adequado de pé diabético aumenta a ocorrência de complicações e a necessidade de amputação; o aumento do número de co-morbidades revela aumento do número de amputações em sujeitos com até três amputações $^{(8)}$.

Ações em saúde, efetivas, no cuidado com os pés, visando à prevenção do pé diabético poderiam evitar 44\% a $85 \%$ das amputações $^{(9,10)}$. Soma-se a isto o estímulo ao autocuidado, o atendimento interdisciplinar e a educação em saúde.

Tal Quadro tem repercussão nos âmbitos sociais, expressos na incapacidade e perda de independência; biológico pelo Quadro de morbidade; psicológico em Que pode comprometer a Qualidade de vida e financeiro, Que por vezes, ocorrem faltas no trabalho e até perda de emprego pela incapacidade gerada. Por outro lado, observam-se as repercussões nos serviços de saúde, através dos custos elevados e das internações prolongadas $^{(4,7)}$.

Evidenciou no estudo realizado na Escócia Que, sujeitos com diabetes tipo II estão associados com admissões mais freqüentes e mais longas Quando comparados com sujeitos não diabéticos, devido as causas neurológicas, cardiovasculares, renais e oftálmicas ${ }^{(11)}$.

Partindo das reflexões sobre a susceptibilidade em Que se encontra a população portadora de diabetes, Questionamos o percentual de sujeitos Que estão sendo submetidos à amputação no Hospital de Clínicas da Universidade Federal do Triângulo Mineiro (UFTM), assim como suas principais características sóciodemográficas e clínicas.
Diante de estudos que identificam características dos sujeitos submetidos à amputação, Questionamos a relação entre amputações e outras variáveis clínicas de interesse no contexto da atenção ao diabético, como o tipo de tratamento, tempo de internação, diagnóstico dentre outros. Desta forma, este estudo busca encontrar respostas à estas Questões com a finalidade de contribuir para o planejamento da atenção ao sujeito diabético e a adęuada atenção aos fatores de riscos para o desenvolvimento de úlceras nos pés, visando prevenir ou retardar seu aparecimento, e, através da análise das variáveis estudadas, auxiliar na implementação de medidas necessárias para mudar a realidade desses indivíduos.

Os objetivos deste estudo foram: descrever as características sócio-demográficas e clínicas de sujeitos internados no Hospital de Clínicas da UFTM Que foram submetidos à amputação relacionada ao diabetes mellitus, no período de 2000 a 2005; comparar o número de amputações segundo: sexo, faixa etária, co-morbidades e tipo de tratamento e associar o número de amputações com o tempo de internação e de diagnóstico.

\section{MÉTODO}

Estudo retrospectivo realizado no Hospital de Clínicas da UFTM.

Os dados foram coletados, através de formulário, nos prontuários de clientes internados no período de 2000 a 2005, Que foram submetidos à amputação cirúrgica relacionada ao diabetes mellitus. A partir da lista do centro cirúrgico, verificou-se a ocorrência de 573 amputações cirúrgicas, das Quais 264 não estavam relacionadas ao diabetes. Em 101 prontuários não foi possível realizar a consulta, visto Que estavam incompletos (falta de volumes) ou não disponíveis no Serviço de Arquivo Médico, no arquivo de óbitos ou tiveram destino desconhecidos. Foram incluídos todos os sujeitos submetidos à amputação com diagnóstico médico de diabetes mellitus registrado no prontuário, no período deste estudo. Portanto, fizeram parte deste estudo 141 sujeitos Que realizaram 208 amputações relacionadas ao diabetes mellitus.

As variáveis estudadas foram: socio-demográficas (idade, sexo, procedência, estado civil, escolaridade, profissão e renda); clínicas (causa de internação primária e secundária, setor de internação, tempo de internação, tempo de diagnóstico de diabetes mellitus, comorbidades, média do nível de glicemia capilar durante a hospitalização, tipo de tratamento para diabetes mellitus, número e região de amputação).

Foi realizado estudo piloto para verficar a adequação do instrumento aos objetivos propostos. Observou-se Que não constava nos prontuários, utilizados para o estudo piloto, dados sobre a escolaridade e renda, no entanto, optou-se por manter esta variável em decorrência de sua importância para o planejamento das ações em saúde. A coleta dos dados foi realizada no período de julho a outubro de 2005.

A glicemia capilar foi classificada de acordo com valores estabelecidos pela Sociedade Brasileira de Diabetes, ou seja, a glicemia de jejum corresponde a $100 \mathrm{mg} / \mathrm{dL}$ e ao acaso menor Que $140 \mathrm{mg} / \mathrm{dL}^{(12)}$.

Os dados foram analisados utilizando-se de medidas descritivas: média e desvio padrão; mediana, valores máximo e mínimo e teste Qui-Quadrado, sendo considerado significativo Quando $p<0,05$.

Esta pesquisa atende a Resolução no 196/96 do Conselho 
Nacional de Saúde e foi aprovada pelo Comitê de Ética em Pesquisa com Seres Humanos da UFTM. Garantiu-se o sigilo e o anonimato dos dados, através da identificação dos formulários por números.

\section{RESULTADOS E DISCUSSÃO}

\section{Características sócio-demográficas e clínicas}

Houve um percentual maior de sujeitos do sexo masculino $(58,9 \%)$ do Que do feminino $(41$, 1\%). A maior ocorrência no sexo masculino corrobora com outros estudos realizados no Brasil, porém isto não está claramente definido na literatura ${ }^{(3,6)}$. Este fato também pode estar relacionado ao maior autocuidado realizado pelas mulheres, possibilitando assim prevenir os fatores de risco relacionados às amputações decorrente do diabetes mellitus.

A mediana da idade foi de 66,5 anos, variando de 24 a 100 anos. A maior ocorrência de amputação está entre os idosos (70,2\%), reforçando os achados em outros estudos Que referem média de 64,8 anos e descrevem maior percentual de amputação entre 61 e 80 anos $(68,7 \%)$, caracterizando a evolução crônica do diabetes ${ }^{(3,6)}$.

A maioria dos sujeitos reside no município de Uberaba (71,7\%), seguindo-se aos de Frutal (4,3\%), Araxá (2,9\%), Campo Florido, São Gotardo e Perdizes (2,2\%) e outros (14,3\%). Destaca-se que o Hospital de Clínicas da UFTM, situado em Uberaba, é referência para a macro-região de saúde do Triângulo Sul, local em Que localizam os referidos municípios.

Quanto ao estado civil, 50,7\% eram casados ou morvamam com companheiro; 17,1\% separados/divorciados/deseuitados/viúvos; $17,1 \%$ nunca se casaram ou moraram com companheiro e $15 \%$ ignorado. Os maiores percentuais obtidos possibilitam Que o profissional de saúde estimule o apoio familiar, identificando problemas culturais, sociais e econômicos Que estejam dificultando a aderência ao tratamento.

Em 99,3\% dos prontuários não constavam à escolaridade e em 100\% não estava mencionada a renda. Para a realização de atividades educativas eficazes, de forma a adequá-la ao entendimento do cliente e contribuir com a adesão ao tratamento, evitando as amputações e incapacidades, a informação sobre a escolaridade é fundamental. Pesquisa realizada no Quênia, evidenciou maior ocorrência de amputações em sujeitos com baixa escolaridade, na Qual 89,1\% tinham educação primária ou nenhuma educação formal, sendo $17,5 \%$ relacionadas ao diabetes mellitus ${ }^{(13)}$.

No Que se refere a renda, tal dado pode contribuir para entender os fatores dificultadores da adesão ao tratamento. Estudo realizado com sujeitos diabéticos, africano-americanos, hispânico-americanos, e outros, mostraram diferenças em taxas de amputação relacionada a pobreza familiar, representando $37,0 \%, 19,0 \%$, e 44,0\%, respectivamente ${ }^{(14)}$.

Estes dados reforçam a necessidade de sensibilização de profissionais de saúde e estudantes para o registro da escolaridade e renda, visto sua relevância para o planejamento e reorganização de ações em saúde. É mister implementar na formação profissional a discussão e reflexão do cuidado ao portador de diabetes mellitus, bem como a importância do registro das informações sobre o cliente, nos prontuários, para Que se possa reorganizar a atenção à saúde através do cuidado direcionado à população em estudo. Destacase, também, a necessidade de sensibilizar os profissionais de saúde para a vulnerabilidade do diabético, visando postergar e prevenir as complicações decorrentes desta doença, em especial o pé diabético que leva a incapacidades e diminui a Qualidade de vida.

A profissão também não estava registrada em 48,2\% dos prontuários, o Que demonstra certa falta de padronização no seu preenchimento. Entre aQueles Que constavam, eram aposentados $(25,5 \%)$; dona de casa $(13,5 \%)$; trabalhador braçal $(2,1 \%)$; não trabalham $(2,1 \%)$; comerciante/bancário $(1,4 \%)$; empregada doméstica $(0,7 \%)$ e outras profissões $(6,4 \%)$. Estes dados necessitariam de maior aprofundamento, não sendo possível, neste momento, pela sua inexistência no prontuário clínico. Contudo, sendo a amostra populacional constituída, em sua maioria por idosos, esperava-se maior proporção de aposentados. No estudo realizado por Awori e Atinga ${ }^{(13)}$ em Que a média de idade da população foi 44,8 anos, verificou Que 55,4\% estavam desempregados e $39,0 \%$ trabalhavam no setor informal.

As causas de internação primária foram: pé diabético $(35,7 \%)$; gangrena (12,7\%); necrose em membro inferior ( $11,2 \%)$; doenças vasculares $(9,9 \%)$; infecção $(9,5 \%)$; lesões $(6,3 \%)$; diabetes mellitus (5\%), doenças renais $(2,1 \%)$ e outros $(7,6 \%)$. Estudo retrospectivo realizado com 288 sujeitos submetidos à amputação maior no Rio Grande do Sul, mostrou Que a causa mais freeüente de amputações estava relacionada à lesão trófica $(68,4 \%)$ e o pé diabético respondeu por $17,3 \%$ das amputações unilaterais ${ }^{(15)}$.

Dentre as causas de internação secundária obteve-se: insuficiência arterial crônica (29,4\%); hipertensão arterial (29,4\%); insuficiência cardíaca $(11,8 \%)$ e outras $(29,4 \%)$. Ressalta-se esta informação constava em 12,1\% dos prontuários.

O diabetes mellitus representa, dentre os diagnósticos primário, a sexta causa de internação hospitalar. Apresenta, ainda, percentuais elevados (30\% a 50\%) para a internação por outras causas como: cardiopatia isquêmica, insuficiência cardíaca, colecistopatias, acidente vascular cerebral e hipertensão arterial ${ }^{(12)}$.

Os setores em Que os sujeitos foram internados eram a clínica cirúrgica $(80,1 \%)$, clínica médica $(9,9 \%)$ e ortopedia $(1,4 \%)$. Em estudo realizado no México, verificou-se que $80,0 \%$ dos leitos destinados a clientes com problemas vasculares são ocupados por sujeitos diabéticos com complicações isQuêmicas e infecciosas do pé(16).

A mediana do número de internação foi um, variando de uma a sete internações. Os maiores percentuais de internação (75\%) foram para uma a duas internações. A mediana do número de dias de internação foi nove, com variação de um a 117 dias. Destes sujeitos, 75,0\% permaneceram internados por até 21 dias. Destaca-se Que $39,3 \%$ dos sujeitos não apresentaram hospitalização anterior. Em decorrência das complicações agudas e, em especial, crônicas do diabetes mellitus, cerca de $14,0 \%$ da amostra é hospitalizada anualmente, com uma média de seis semanas para cada internação(6).

Aproximadamente, 15,0\% dos clientes com diabetes desenvolverão úlceras de pé e 6,0\% serão hospitalizados devido à essa complicação ${ }^{(17)}$. Em estudo de coorte foi observado Que o risco de hospitalização era 55,7 vezes maior em sujeitos diabéticos Que desenvolveram infecção nos pés do Que os Que não desenvolveram. Observou-se Que a infecção nos pés contribuiu para hospitalização em $71,7 \%$ dos casos e o risco de amputação foi 154,5 vezes maior ${ }^{(18)}$.

A mediana de tempo de diagnóstico do diabetes mellitus foi de 12 anos, com variações de menor Que um ano a 40 anos; $40,9 \%$ dos 
sujeitos receberam diagnóstico entre 10 a 20 anos anteriores à última amputação. Estes resultados corroboram com a literatura, em Que se observou mediana de 10 anos, com amplitude de zero a 40 anos ${ }^{(19)}$.

Verificou-se que 90, 1\% dos sujeitos apresentam co-morbidades, distribuídas numericamente em: uma morbidade $(29,8 \%)$; duas $(31,2 \%)$, três $(17,0 \%)$; Quatro $(9,3 \%)$, cinco $(2,1 \%)$ e seis $(0,7 \%)$.

As co-morbidades mais freeüentes foram: hipertensão arterial (74\%), tabagismo (22\%), problemas cardíacos (19,9\%), retinopatia $(17,8 \%)$, aterosclerose $(14,9 \%)$, alcoolismo $(9,2 \%)$, nefropatia $(5 \%)$ e neuropatia (2,8\%). A hipertensão arterial (91,2\%), também, foi a doença mais encontrada no estudo realizado com 56 casos de pé diabético avaliados no Hospital Universitário de Mato Grosso do $\mathrm{Sul}^{(8)}$. Os autores reforçam Que o diabetes mellitus e a hipertensão arterial, em conjunto, aumentam os fatores de risco para doenças micro e macrovasculares, contribuindo inclusive para a mortalidade cardiovascular $^{(8)}$.

A retinopatia diabética $(3,2 \%)$, a cegueira $(1,6 \%)$ e a insuficiência renal $(8,8 \%)$ também foi identificada em estudo realizado com sujeitos diabéticos ${ }^{(20)}$

Questionamos se os sujeitos deste estudo, em sua maioria hospitalizados por até duas vezes, apresentando pelo menos duas co-morbidades, estão sendo devidamente acompanhados pelos serviços de atenção primária. Apesar dos diversos fatores Que influenciam na adesão ao tratamento, espera-se fornecer atenção multiprofissional com acompanhamento e apoio domiciliar de modo a promover saúde e bem-estar.

A mediana do nível glicêmico, no período da última internação, foi de $142,2 \mathrm{mg} / \mathrm{dL}$, com valores mínimo de $66,9 \mathrm{mg} / \mathrm{dL}$ e máximo $342,8 \mathrm{mg} / \mathrm{dL}$. Destaca-se Que os índices glicêmicos, ao acaso, dentro da normalidade (menor Que $140 \mathrm{mg} / \mathrm{dL}$ ) foram mantidos por $44,7 \%$ dos sujeitos, durante a internação. Em 4,9\% dos prontuários não continham essa informação. Estudos tem evidenciado Que sujeitos diabéticos Que realizam rigoroso controle glicêmico, tem apresentado menores índices de complicações neuropáticas ${ }^{(2)}$.

A mediana do número de amputações foi duas, com valores mínimo de uma e máximo de oito, sendo os maiores percentuais de amputações por sujeito: uma $(48,2 \%)$, duas $(28,4 \%)$ e três (15,6\%). Estudos descrevem taxas cumulativas de reamputação de $26,7 \%$ em um ano, $48,3 \%$ em três anos e $60,7 \%$ em cinco anos ${ }^{(22)}$ e número médio de amputações de $1,6^{(23)}$.

Os pés de sujeitos com diabetes devem ser examinados por profissionais de saúde pelo menos uma vez ao ano, e na presença de fatores de risco, a cada um a seis meses ${ }^{(8)}$. Estudo prospectivo realizado com sujeitos portadores de neuropatia evidenciou Que 54,0\% das úlceras eram devido trauma causado pelos calçados ${ }^{(24)}$. Estes dados reforçam a implementação do exame nos pés nos serviços de saúde, em especial, nos de atenção primária, local privilegiado para a realização da promoção da saúde e prevenção de doenças.

As medidas preventivas são essenciais na assistência ao pé diabético, o controle glicêmico, a educação em saúde sobre formas de evitar ulcerações, o cuidado adeQuado dos pés e visitas regulares ao profissional de saúde, assim como a colaboração do cliente e familiares são fundamentais na prevenção de incapacidades e deformidades por pé diabético ${ }^{(8)}$.

Cabe aQui uma reflexão sobre até Que ponto os serviços de saúde estão contribuindo com tal situação, na medida em Que os clientes comparecem aos serviços e, na maioria das vezes, não tem seus pés inspecionados e poucos são estimulados a participarem de atividades educativas. E Quando são, Que contribuições essas atividades estão oferendo aos seus clientes, haja vista Que constatamos predomínio de internações por pé diabético e tempo médio de diagnóstico de 12 anos.

O tratamento instituído no momento da internação foi: hipoglicemiante oral $(55,7 \%)$; insulina $(24,6 \%)$, hipoglicemiante oral e insulina $(14,4 \%)$ e não medicamentoso $(5,2 \%)$. Em 31,2\% dos prontuários não constava esse dado. $\mathrm{O}$ tratamento do diabetes requer mudança de hábitos, realização de atividades físicas, uso de medicamentos, Quando necessário, e educação em saúde. Brasileiro et $\mathrm{al}^{(8)}$ referem Que dos sujeitos portadores de pé diabético Que realizavam tratamento $(83,9 \%), 61,7 \%$ utilizavam hipoglicemiantes orais, $34 \%$ insulina, e 4,3\% insulina e hipoglicemiantes orais associadamente. Na prevenção do pé diabético a adesão ao tratamento é essencial, constituindo a educação em saúde um espaço privilegiado para contribuir com a adesão ao tratamento do diabetes ${ }^{(25)}$.

O intervalo de tempo entre as amputações foi em 71,2\% dos casos menor Que seis meses. Os percentuais no número de amputações representou: duas amputações (64,7\%); três (81,3\%); Quatro $(72,7 \%)$ e cinco $(85,7 \%)$. Não constava intervalo de tempo entre a primeira e a segunda amputação em $6,8 \%$ dos prontuários. Estes dados demonstram Que independente do número de amputações a maioria dos sujeitos tem intervalo curto entre as amputações evidenciando a dificuldade de recuperação do membro e a necessidade de re-amputação. Esses achados são consoantes com o estudo desenvolvido com informações de 4.818 amputações em sujeitos com doença arterial periférica e diabetes mellitus, no período de 1990 a $2000^{(3)}$. Evidenciou-se Que, a maioria das recorrências de amputação ocorreram em um intervalo menor Que 6 meses e Que, somente, 25,0\% dos sujeitos amputados apresentam pulsos distais (pedioso e tibial posterior) no membro contra-lateral, e estima-se Que, aproximadamente, 15,0\% dos clientes necessitarão de amputação neste membro em dois anos ${ }^{(3)}$. Estudo desenvolvido com sujeitos biamputados evidenciaram Que 49,2\% apresentaram amputação maior contralateral no intervalo inferior a um ano(15).

A localização das amputações foi: infragenicular esquerda $(13,6 \%)$, infragenicular direita $(12,5 \%)$, supragenicular direita $(11,7 \%)$ e primeiro pododáctilo direito $(9,1 \%)$. O local mais freeüente da última amputação foi: supragenicular direita $(18,6 \%)$, seguida de infragenicular esquerda (15,7\%), infragenicular direita $(13,6 \%)$ e supragenicular esquerda (12,9\%). Resultados Que corroboram com a literatura Que evidencia Que a maioria das amputações são suprageniculares $(76,3 \%)$ seguidas de infrageniculares $(23,7 \%)^{(15)}$. Dentre as amputações suprageni-culares, observou-se Que 40, I\% estavam relacionadas ao diabetes mellitus ${ }^{(3)}$.

A evolução mais frequente da hospitalização foi a alta hospitalar (72,3\%) e o óbito (28\%). Porém, estudo realizado no Rio Grande do Sul, revela percentual menor na taxa de mortalidade em grupo de sujeitos submetidos à amputação unilateral $(22,7 \%)$ e maiores entre aQueles com amputação bilateral (50\%), considerando até o $30^{\circ}$ dia de pós-operatório ${ }^{(15)}$. Brasileiro et $a^{(8)}$ refere taxa de $3,6 \%$.

\section{Número de amputações}

Apesar de haver maior percentual de amputações entre os homens $(58,2 \%)$ em relação às mulheres $(41,8 \%)$, não se observou diferença significativa entre os $\operatorname{sexos}\left(x^{2}=5,25 ; p=0,073\right)$. 
Resultados semelhantes foram encontrados em outros estudos realizados no Rio de Janeiro ${ }^{(3)}(57,9 \%)$ e em Alagoas $^{(6)}(57,2 \%)$. O maior número de amputações no sexo masculino pode estar relacionado ao maior cuidado do sexo feminino com os pés, assim como sua maior presença nos serviços de saúde, Que pode favorecer o diagnóstico precoce da úlcera nos pés.

As faixas etárias Que apresentaram maior percentual Quanto ao número de amputação foram de 70 a 80 anos $(37,5 \%)$ e 60 a 70 anos (26,9\%), não sendo observado diferenças significativas entre as faixas estudadas $\left(x^{2}=3,99 ; p=0,68\right)$. Maior ocorrência de amputação em pessoas com idade mais avançada foi verificado também em outros estudos. Observou-se aumento da incidência de amputações nos membros inferiores, relacionadas ao diabetes mellitus, de 17,8 vezes maior para a faixa etária de 30 a 89 anos e 19 vezes maior para a de 55 a 74 anos $^{(3)}$. Verificou-se, também, a ocorrência Que a amputação foi, significativamente, mais freQüente na faixa etária de 60 a 90 anos, se comparada à de 40 a 60 anos $^{(19)}$.

Não se obteve associação significativa entre o número de amputações e a presença de co-morbidade $\left(x^{2}=6,26 ; p=0,62\right)$. Os maiores percentuais de sujeitos amputados foram para aQueles Que possuíam uma morbidade $(30,5 \%)$ e duas $(30,5 \%)$. Dentre aqueles Que realizaram uma amputação $(32,4 \%)$ e três $(31,8 \%)$ a maioria tinha uma morbidade, já entre os Que tiveram duas $(32,5 \%)$ e de Quatro a oito amputações $(45,5 \%)$ possuíam duas co-morbidades. Estudo retrospectivo Que objetivou determinar os resultados de uma série de amputações contemporâneas, destacaram-se as comorbidades: diabetes mellitus $(49,4 \%)$, tabagismo $(81,6 \%)$, hipertensão arterial $(77,0 \%)$ e doença is@uêmica do coração $(58,6 \%)$ (26). Ademais, a demora no início do tratamento adequado do pé diabético aumenta a ocorrência de complicações e, consequentemente, a necessidade de amputação ${ }^{(25)}$.

Ao analisar o número de amputações realizadas com o tipo de tratamento, não se verificou associação significativa $\left(x^{2}=4,60\right.$; $p=0,59)$. Independente do número de amputações realizadas, todos os maiores percentuais foram para aqueles sujeitos Que fazem uso de hipoglicemiante oral. Estudo realizado com sujeitos portadores de pé diabético submetidos à amputação também evidenciou Que a maioria utilizava hipoglicemiante oral $(55,0 \%)$ e insulina $(20 \%)^{(8)}$. Este dado sugere a necessidade de maior aprofundamento sobre a adesão ao tratamento medicamentoso e não medicamentoso, visando verificar possíveis fatores de risco para o desenvolvimento do pé diabético.

\section{Tempo de internação}

Não houve associação significativa no tempo de internação de acordo com o número de amputação $\left(x^{2}=12,80 ; p=0,24\right)$. Os maiores percentuais para aQueles Que realizaram uma amputação $(54,4 \%)$, duas $(50 \%)$, três $(36,7 \%)$ e Quatro a oito $(71,4 \%)$ permaneceram internados até 10 dias. Estudo retrospectivo que analisou a média de duração de hospitalização, demonstrou maior número de dias entre os sujeitos submetidos a amputação (45) e menor entre os Que não haviam sido amputados $(28,95)^{(27)}$.

O pé diabético está associado a altas taxas de ocupação e permanência hospitalar, com repetidas intervenções cirúrgicas, nem sempre compatível com a tabela do SUS. Além disso, há de se considerar o custo social, traduzido nas faltas do trabalho, nas aposentadorias precoces e no custo para o cliente diabético Que tem a sua Qualidade de vida comprometida ${ }^{(8)}$.

Apesar do tempo de diagnóstico ser um dos fatores de risco para o aparecimento do pé diabético, nestes achados não se verificou sua associação significativa com o número de amputações $\left(x^{2}=9,05\right.$; $p=0,06)$. Dentre os sujeitos com uma amputação, a maioria $(50,0 \%)$ teve diagnóstico do diabetes mellitus em até 10 anos; com duas amputações $(48,4 \%)$ e três $(54,5 \%)$ entre 1 I a 20 anos e com Quatro a oito $(57,1 \%) 21$ a 30 anos. Estes dados corroboram com a literatura Que não evidencia relação entre a amputação e tempo de diagnóstico. O tempo médio de diagnóstico da referida doença é de 16 anos, momento em Que ocorre o maior percentual de amputações ${ }^{(27,28)}$. Durante 8,7 anos de acompanhamento de sujeitos diabéticos, observou-se Que $26 \%$ dos Que foram submetidos à amputação em extremidade inferior morreram devido doença cardiovascular sendo média de duração do diabetes mellitus de 19,8 anos ${ }^{(29) \text {. }}$

Considerando a complexidade Que envolve o tratamento do diabetes mellitus, a adesão do cliente ao tratamento será favorecida através de sua participação efetiva e, em conjunto com os profissionais de saúde e familiares. Deste modo, o tratamento deve também observar a motivação pessoal, aceitação da doença e apoio familiar visando postergar as amputações e promover melhoria da Qualidade de vida ao sujeito diabético ${ }^{(30)}$.

\section{CONCLUSÕES}

Este estudo evidencia Que a maioria dos sujeitos submetidos à amputação é do sexo masculino, idosos, com tempo de diagnóstico de 10 a 20 anos, fazendo uso de hipoglicemiante oral, apresentando, em média, níveis glicêmicos acima do padronizado, com períodos prolongados de internação e com menos de seis meses entre as amputações.

Não foram observadas diferenças significativas entre número de amputações e sexo, faixa etária, co-morbidades e tipo de tratamento. Também não houve associação significativa no tempo de internação e tempo de diagnóstico de acordo com o número de amputações.

\section{REFERÊNCIAS}

I. Secretaria de Estado de Saúde do Distrito Federal. Consenso Internacional sobre pé diabético. Brasília: Imprensa Oficial; 1999.

2. Caiafa IS, Canongia PM. Atenção integral ao paciente com pé diabético: um modelo descentralizado de atuação no Rio de Janeiro. J Vasc Br 2003; 2(1): 75-8.

3. Spichler D, Miranda Ir F, Spichler ES, Franco LJ. Amputações maiores de membros inferiores por doença arterial periférica e diabetes melito no município do Rio de Janeiro. I Vasc Br 2004;

\section{3(2): III-22.}

4. Calsolari MR, Castro RF, Maia RM, Maia FCP, Castro AV, Reis R et al. Análise retrospectiva dos pés de pacientes diabéticos do ambulatório de diabetes da Santa Casa de Belo Horizonte, MG. Are Bras Endocrinol Metab 2002; 46(2): 173-6.

5. Scheffel RS, Bortolanza D, Weber, CS, Costa WLA, Canani LH, Santos KG, et al. Prevalência de complicações micro e macrovasculares e seus fatores de risco em pacientes com 
diabete melito do tipo 2 em atendimento ambulatorial. Rev Assoc Med Bras 2004; 50(3):263-7.

6. Pitta GBB, Castro AA, Soares AMMN, Maciel CII, Silva JDM, Muniz VMT, et al. Perfil dos pacientes portadores de pé diabético atendidos no Hospital Escola José Carneiro e na Unidade de Emergência Armando Lages. I Vasc Br 2005; $4(1): 5-10$.

7. Lluveras ILS, Domínguez IM. Guia práctica para el diagnóstico y el tratamiento del síndrome del pie diabético. Rev Cubana Endocrinol 200I; 12(3): 188-97.

8. Brasileiro JL, Oliveira WTP, Monteiro LB, Chen J, Pinho Jr EL, Molkenthin S, et al. Pé diabético: aspectos clínicos. J Vasc Br 2005; 4(1):11-21.

9. Gamba MA. Amputações por diabetes mellitus: uma prática prevenível? Acta Paul Enferm 1998; I I (3):92-100.

10. Pedrosa HC. Pé diabético: aspectos fisiopatológicos, tratamento e prevenção. Rev Bras Neurol Psie 1997; I (3): 13 I-35.

11. Donnan PT, Leese GP, Morris AD. Hospitalizations for people with type I and type 2 diabetes compared with the nondiabetic population of Tayside, Scotland. Diabetes Care 2000; 23(12): 1774-9.

12. Sociedade Brasileira de Diabetes. Consenso Brasileiro Sobre Diabetes 2002: diagnóstico e classificação do diabetes melito e tratamento do diabetes melito do tipo 2. São Paulo: Diagraphic: 2003.

13. Awori KO, Atinga JE. Lower limb amputations at the Kenyatta National Hospital, Nairobi. East Afr Med I 2007; 84(3): 12 I 6.

14. Wachtel MS. Family poverty accounts for differences in lowerextremity amputation rates of minorities 50 years old or more with diabetes. I Natl Med Assoc 2005; 97(3): 334-8.

15. Leite CF, Frankini AD, DeDavid E, Haffner I. Análise retrospectiva sobre a prevalência de amputações bilaterais de membros inferiores. I Vasc Br 2004;3(3):206- I3.

16. Medina E, Villena A. Características clínicas de pie diabético. Bol Soc Peruana Med Interna 2000:3-7.

17. Bloomgarden ZT. American Diabetes Association 60th Scientific Sessions, 2000: the diabetic foot. Diabetes Care 2001; 24(5): 946-5.

18. Lavery LA, Armstrong DG, Wunderlich RP, Mohler MJ, Wendel CS, lipsky BA. Risk factors for foot infections in individuals with diabetes. Diabetes Care 2006; 29(6): 1288-93.

19. Nunes MAP, Resende KF, Castro AA, Pitta GB. Fatores predisponentes para amputação de membro inferior em pacientes diabéticos internados com pés ulcerados no estado de Sergipe. I Vasc Br 2006;5(2): 123-30.

20. Grillo MFF, Gorini MIPC. Caracterização de pessoas com Diabetes Mellitus Tipo 2. Rev Bras Enferm 2007; 60(I): 49-54.

21. Nascimento OIM. Neuropatia diabética: diagnóstico e tratamento. In: Oliveira JEP, Milech A, organizadores. Diabetes mellitus: clínica, diagnóstico, tratamento multidisciplinar. São Paulo: Atheneu; 2004. p 183-97.

22. Izumi Y, Satterfield K, Lee S, Harkless LB. Risk of reamputation in diabetic patients stratified by limb and level of amputation: a 10-year observation. Diabetes Care 2006; 29(3): 566-70.

23. Bilenko V, Bilenko N, Harman-Boehm I, Atar D, Rosen S, Weitzman S. Trends and characteristics of diabetes-related lower limb amputations in the Negev, 1996-1999. Harefuah 2006; 145(10): 709-12.

24. McGill M, Molyneaux L, Yue DK. Which diabetic patients should receive podiatry care? An objective analysis. Intern Med I 2005; 35(8): 45 I-6.

25. Gamba MA, Gotlieb SLD, Bergamaschi DP, Viana LAC.Amputações de extremidades inferiores por diabetes mellitus: estudo caso-controle. Rev Saúde Pública 2004; 38(3): 399-404.

26. Lim TS, Finlayson A, Thorpe JM, Sieunarine K, Mwipatayi BP, Brady A, et al. Outcomes of a contemporary amputation series. ANZ I Surg 2006; 76(5): 300-5.

27. Karakoc A, Ersoy RU, Arslan M, Toruner FB, Yetkin I. Change in amputation rate in a Turkish diabetic foot population. I Diabetes Complications 2004; 18(3): 169-72.

28. Real-Collado IT, Valls M, Basanta-Alario ML, Ampudia-Blasco FI, Ascaso-Gimilio JF, Carmena-Rodríguez R. Estudio de factores asociados con amputación, en pacientes diabéticos con ulceración en pie. An Med Interna 200 I; I 8(2): 13-8.

29. Resnick HE. Relation of lower-extremity amputation to all-cause and cardiovascular disease cortality in American indians. The Strong Heart Study. Diabetes Care 2004; 27: 1286-93.

30. Santos ECB, Zanetti ML, Otero LM, Santos MA. O cuidado sob a ótica do paciente diabético e de seu principal cuidador. Rev Latino-am Enfermagem 2005; 13(3): 397-406. 